Meta

Journal des tradlucteurs

Translators' Journal

\title{
Moeschler, Jacques et Anne Reboul (1994) : Dictionnaire encyclopédique de pragmatique, Paris, Éditions du Seuil, 562 p.
}

\section{Marty Laforest}

Volume 40, numéro 4, décembre 1995

URI : https://id.erudit.org/iderudit/003373ar

DOI : https://doi.org/10.7202/003373ar

Aller au sommaire du numéro

Éditeur(s)

Les Presses de l'Université de Montréal

ISSN

0026-0452 (imprimé)

1492-1421 (numérique)

Découvrir la revue

Citer ce compte rendu

Laforest, M. (1995). Compte rendu de [Moeschler, Jacques et Anne Reboul (1994) : Dictionnaire encyclopédique de pragmatique, Paris, Éditions du Seuil, 562 p.] Meta, 40(4), 677-680. https://doi.org/10.7202/003373ar d'utilisation que vous pouvez consulter en ligne.

https://apropos.erudit.org/fr/usagers/politique-dutilisation/ 
MOESCHLER, Jacques et Anne REBOUL (1994): Dictionnaire encyclopédique de pragmatique, Paris, Éditions du Seuil, 562 p.

Voila un ouvrage important et attendu, qui comble un besoin: celui de voir regroupé en un tout cohérent un ensemble de recherches menées essentiellement en Europe francophone el depuis une vingtaine d'années en pragmatique. science de l'usage du langage.

Jacques Moeschler et Anne Reboul étaient on ne peut mieux placés pour accomplir ce travail. Le premier, auteur de plusieurs ouvrages dans le domaine, faisait déjà partie du groupe dit de Genève à sa fondation par Eddy Roulet au début des années quatre-vingts : la seconde, bien connue pour ses travaux en stylistique, est docteur en linguistique et en philosophie et a été formée par Oswald Ducrot. Tous deux sont partie prenante de ces recherches auxquelles ils ont apporté une contribution importante. et se situent au cœur de la mouvance créé autour des travaux genevois, des théories d'Oswald Ducrot, de Dan Sperber et Deirdre Wilson, de Gilles Fauconnier, etc. D'entrée de jeu, ils donnent le désormais classique Dictionnaire encyclopédique des sciences du langage de Ducrot et Todorov (1972) comme le modèle qui a inspiré leur démarche. Toutefois, le projet initial a évolué de telle sorte que le produit final ne se présente pas comme une suite alphabétique de rubriques consacrées à des points précis, à l'instar du livre de Ducrot et Todorov, mais comme un recueil d'articles de synthèse sur les grandes questions auxquelles la pragmatique a pour objectif de répondre. Une part importante de l'originalité et de l'utilité de l'ouvrage tient d'ailleurs dans sa construction : les chapitres sont autonomes, mais 
liés : on peut lire ce dictionnaire d'un bout à l'autre dans l'ordre, le tout constituant une sorte de séminaire de pragmatique abordable pour le néophyte éclairé comme pour celui qui a un peu pataugé dans le domaine mais a qui manque justement cette cohérence, ces liens d'une théorie et d'un concept à l'autre. Mais on peut également lire chaque chapitre séparément si on veut faire le point sur un problème précis : en cela, le Dictionnaire est vraiment l'ouvrage de référence pour les pragmaticiens plus avancés, qui bénéficieront de la finesse des analyses, de la subtilité autant que de la précision des distinctions apportées. Reste, dans tous les cas de figure, le plaisir de la consultation : l'aridité (parfois) de la matière est contrebalancée par le réel souci de clarté qui semble avoir motivé lés auteurs.

Le dictionnaire de pragmatique de Moeschler et Reboul s'ajoute à un certain nombre d'autres ouvrages également consacrés au cours des dernières années à l'étude du langage en contexte envisagé dans toute son étendue (voir Levinson (1983), qui est certainement l'un des plus connus, mais aussi notamment Latraverse (1987), Mey (1993) et Blakemore (1992)). Sauf Latraverse (1987), qui est un ouvrage très philosophique, la plupart ont une visée pédagogique explicite, et sont accompagnés, suivant une tradition éditoriale anglo-saxonne bien établie, d'exercices en fin de chapitres à l'intention des étudiants. Moeschler et Reboul ont conçu leur livre de façon très différente. Bien qu'il soit relativement accessible au non-spécialiste, il témoigne d'une ambition plus grande : celle d'occuper un créneau théorique précis, de renforcer la position du courant ouvert par la théorie de la pertinence de Sperber et Wilson (1986).

Ce faisant, Reboul et Moeschler évitent l'écueil de l'éclatement, danger auquel certains auteurs n'échappent que difficilement. Ce n'est en effet qu'au prix de la restriction du terrain théorique couvert que la cohérence peut être atteinte. La pragmatique $n^{\prime}$ 'a en fait véritablement pris son essor qu'il y a une trentaine d'années et elle est encore en quête de son autonomie par rapport aux disciplines qui se l'approprient plus ou moins directement (la source de la pragmatique est principalement philosophique, mais actuellement. la pragmatique touche à la linguistique, à l'analyse du discours, à l'anthropologie, à la psychologie, voire à la sociologie et même aux études littéraires). La juxtaposition hétéroclite des traditions. des postulats et des objectifs de l'ensemble des courants qui l'alimentent ou s'en réclament aujourd'hui eût été peu conciliable avec les impératifs d'une véritable démarche scientifique.

L'ouvrage occupe donc une position précise dans l'univers conceptuel de la discipline : si les auteurs mentionnent dès l'introduction que la pragmatique est avant tout un champ anglo-saxon et que les exceptions sont rares sur le continent européen, c'est pour consacrer à ces exceptions la plus grande partie de l'ouvrage. Il ne s'agit pas de LA pragmatique, mais d'une pragmatique, celle de Moeschler et Reboul, qui est élaborée sur la base de la théorie de la pertinence et se situe done sur le terrain de la psychologie cognitive. Pour Moeschler et Reboul comme pour Sperber el Wilson. les mécanismes en jeu dans l'interprétation des énoncés ne sont pour l'essentiel pas différents de ceux qui régissent les autres champs d'activité humaine exigeant la compréhension. La pragmatique mise de l'avant par les auteurs du dictionnaire doit aussi beaucoup à Ducrot et pone également la trace de la linguistique de Milner.

Dans la première partie de l'ouvrage sont abordés le statut de la pragmatique et la question de son rattachement aux sciences du langage, qui revient à celle du rapport entre la structure linguistique (phonologie, syntaxe, sémantique) et les emplois qu'on peut en faire. La manière Moeschler/Reboul de poser les problèmes apparaît d'emblée par la netteté et la rigueur du raisonnement : les conséquences et les implications de chaque option théorique sont discutées et rassemblées en une synthèse assez éblouissante. C'est aussi dans cette partie de l'ouvrage qu'est présentée la théorie classique des actes de langage. 
Les chapitres suivants sont directement liés au «programme» de la pragmatique, celle-ci s'étant donné pour tâche d'identifier les processus en jeu dans la détermination du sens des énoncés. Cette interprétation des énoncés suppose l'identification des référents et le choix du sens approprié en cas d'ambiguité au niveau de la forme linguistique (ambiguîté lexicale, syntaxique, etc.). Les différents aspects de ces questions sont traités en détail et les auteurs démontrent bien que des problèmes traditionnellement considérés comme relevant de la sémantique - la référence est de ceux-la - reçoivent un traitement beaucoup plus satisfaisant dans le cadre pragmatique. La notion de contexte détient à cet égard un rôle central, longuement débattu.

Le sens implicite - ou implicature - fait l'objet d'un deuxième grand groupe de chapitres : d’amples développements sont consacrés aux connecteurs, aux lois de discours, à la présupposition, aux théories de l'argumentation et de l'énonciation de Ducrot ainsi qu'a la notion de polyphonie qu'il a développée, de même qu'à la question des anaphoriques et des déictiques - autre aspect du problème de la référence. Enfin, le traitement pragmatique de questions d'ordre stylistique est abordé, notamment la question de la métaphore et le statut du discours fictionnel. Les deux derniers chapitres traitent des liens qu'entretient l'analyse du discours avec la pragmatique. L'analyse de discours est ici envisagée presque uniquement dans sa perspective structurale: on y présente le modèle hiérarchique genevois et on y traite de la cohérence thématique et de la progression temporelle.

La question de la relation entre discours et pragmatique $m$ 'apparaît fondamentale et elle est un peu rapidement évacuée. Le discours représente l'épreuve du réel langagier et l'interprétation des énoncés est largement liée à leur énonciation en séquences dialogales ou non. Mais, fidèles à leur parti pris pour un usage plutôt idéalisé du langage, Moeschler et Reboul y attachent peu d'importance. ce qui explique la minceur de cette partie de leur ouvrage. Manifestement, l'analyse du discours n'est pas leur tasse de thé : l'élaboration d'un cadre conceptuel prend largement le pas sur la vérification de son adéquation avec la réalité discursive et le terrain des études empiriques est jugé assez peu intéressant. Là s'affrontent explicitement, bien que brièvement, deux épistémologies, deux approches méthodologiques opposées, affrontement dont le fondement tient peutêtre en partie au fait que l'Europe francophone n'a pas de tradition de terrain sur ce plan : certains courants pragmatiques, notamment en Amérique du Nord, ne reposent pas que sur des bases philosophiques et s'appuient également sur la tradition de l'anthropologie linguistique, qui a toujours accordé une grande importance au travail de terrain. $D$ où le développement de toute une pragmatique développementale et expérimentale, appuyée sur des productions réelles de locuteurs réels et bien représentée dans une revue telle que Journal of Pragmatics.

La formulation de cette insatisfaction de la lectrice que je suis de Moeschler et Reboul n'enlève toutefois rien à la qualité de leur déjà indispensable dictionnaire, dont le principal mérite est je crois de faire apparaître la pragmatique, si jeune soit-elle, comme un corps constitué d'un certain nombre d'acquis théoriques. sur lesquels on peut appuyer la recherche à venir. La vision de ce corps constitué de connaissances, difficile à percevoir dans l'hétérogénéité et la fragmentation qui caractérisent la discipline, a quelque chose de fort réjouissant.

MARTY LAFOREST 


\section{REFERENCES}

BLAKEMORE. Diane (1992) : Understanding Utrerances. An Introduction to Pragmatics, Ox ford, Blackwell. DUCROT. Oswald et T'vetan TODOROV (1972): Dictionnaire enc yclopédique des scicmeses du langage. Paris, Seuil.

LATR AVERSE. François (1987) : La pragmanques. Histrire e't critique, Bruxelles, Pierre Mardaga éditeur. LEVINSON, Stephen C. (1983): Pragmuatics. Cambridge, Cambridge University Press.

MEY. Jacob L.. (199.3) : Pragmatics. An Introductiom. Oxford. Blackwell.

MILNER, Jean-Claude (1989): Introductuon d une science du langage. Paris, Seuil.

SPERBER, Dan et Deirdre WILSON (1986): Relevance' Commumication and Cognition, Oxford. Blackwell,

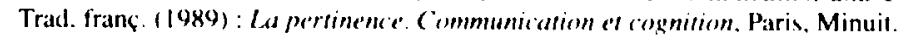

